\title{
Un micro-organisme redoutable à nos portes : ce que les fournisseurs de soins de santé doivent savoir au sujet de Candida auris
}

\author{
IS Schwartz ${ }^{1 *}$, SW Smith, TC Dingle ${ }^{2,3}$
}

\section{Résumé}

Candida auris est un champignon pathogène d'apparition récente qui se répand rapidement dans le monde entier. II est à présent au Canada. C. auris peut causer des maladies invasives associées à un taux de mortalité élevé. Il est souvent résistant à une ou à plusieurs classes d'antifongiques et peut être difficile à identifier dans certains laboratoires de microbiologie clinique. C. auris peut également coloniser la peau des patients durant de longues périodes, contaminer les milieux ambiants et être ainsi à l'origine d'éclosions nosocomiales dans les hôpitaux et les établissements de soins de longue durée.

Les cliniciens, les professionnels de la prévention et de la prise en charge des infections et les responsables de la santé publique doivent savoir comment atténuer la menace posée par ce pathogène. Un cas de référence d'infection par $C$. auris doit être soupçonné chez les patients atteints d'une candidose invasive qui ont récemment été hospitalisés dans des régions du monde où $C$. auris est répandu, ainsi que chez les patients qui ne répondent pas au traitement antifongique empirique et chez qui des espèces non identifiées ou inhabituelles de Candida ont été isolées. Si un cas d'infection ou de colonisation par C. auris est découvert ou soupçonné, les mesures suivantes doivent être prises : déclaration du cas aux autorités locales de santé publique et aux professionnels de la prévention et de prise en charge des infections; placement des patients colonisés ou infectés dans des chambres privées et prise de précautions contre les contacts; désinfection de l'environnement au quotidien et à la fin du séjour au moyen d'un agent sporicide; recherche des contacts et dépistage de la transmission de C. auris; envoi des isolats suspects ou confirmés aux laboratoires provinciaux. Les patients atteints d'une maladie symptomatique doivent être traités au moyen d'une échinocandine en attendant les résultats des épreuves de sensibilité aux antifongiques, de préférence en consultation avec un spécialiste des maladies infectieuses. Grâce à la vigilance des travailleurs de la santé de première ligne et des microbiologistes, aux pratiques rigoureuses de prévention et de prise en charge des infections et aux efforts de surveillance à l'échelle locale et nationale, $C$. auris peut être décelé rapidement, les infections prises en charge et les transmissions prévenues pour protéger les patients de notre système de soins de santé.

\section{Affiliations}

${ }^{1}$ Division des maladies infectieuses, faculté de médecine, Université de l'Alberta, Edmonton (Alberta)

${ }^{2}$ Division de microbiologie diagnostique et appliquée, département de médecine de laboratoire et de pathologie, Université de l'Alberta, Edmonton (Alberta)

${ }^{3}$ Provincial Laboratory for Public Health, Edmonton (Alberta)

*Correspondance :

ilan@ualberta.ca

Citation proposée : Schwartz IS, Smith SW, Dingle TC. Un micro-organisme redoutable à nos portes : ce que les fournisseurs de soins de santé doivent savoir au sujet de Candida auris. Relevé des maladies transmissibles au Canada 2018;44(11):308-14. https://doi.org/10.14745/ccdr.v44i11a01f

Mots-clés : Candida auris, septicémie à Candida, infections fongiques, mycologie, résistance aux antifongiques, multirésistance aux médicaments, diagnostic, nosocomial, prévention et prise en charge des infections

\section{Introduction}

En juillet 2017, le premier cas connu de Candida auris multirésistant a été signalé au Canada chez un sujet qui rapportait des problèmes récurrents aux oreilles depuis deux ans, suivant son retour d'un voyage en Inde, où il avait été hospitalisé à cause d'un abcès du cerveau consécutif à une chirurgie buccale (1). Ce cas a marqué l'arrivée au Canada d'un pathogène qui se répand depuis peu dans le monde entier. La capacité de ce champignon de provoquer une maladie invasive, 
sa résistance fréquente à au moins une classe d'antifongiques et la possibilité démontrée de sa transmission nosocomiale préoccupent autant les cliniciens que les professionnels de la santé publique $(2,3)$.

L'objectif du présent article est de résumer nos connaissances sur ce champignon; de décrire les enjeux liés au diagnostic, au traitement, à la prévention et à la prise en charge des infections; et de cerner les efforts engagés pour surveiller et limiter la propagation de ce pathogène au Canada.

\section{Quels pays sont touchés par $C$. auris?}

C. auris a été décrit pour la première fois au Japon en 2009; depuis lors, des cas d'infections par $C$. auris ont été signalés dans au moins 30 pays répartis sur six continents (4). Des analyses séquentielles du génome entier d'isolats prélevés dans différents pays ont montré que ceux-ci se regroupent en clades géographiques (clonaux) très apparentés (5), ce qui suggère l'émergence quasi simultanée de $C$. auris sur au moins trois continents. Par exemple, les distances génétiques moyennes entre les clades d'Extrême-Orient, d'Asie du Sud, d'Afrique du Sud et d'Amérique du Sud étaient comprises entre 40000 et 140000 polymorphismes mononucléotidiques (single nucleotide polymorphisms [SNP]), alors qu'en moyenne, moins de 70 SNP séparaient deux isolats au sein d'un clade donné (3). Ce phénomène est encore inexpliqué.

Dans certains pays, C. auris a déjà alourdi significativement le fardeau imposé par les maladies nosocomiales. Par exemple, C. auris est la cause de septicémie à Candida dans $10 \%$ des cas à l'échelle nationale en Afrique du Sud (6), et dans $38 \%$ des cas dans un hôpital de référence au Kenya (Okinda, N. et coll. "Candidemia at a referral hospital in sub-Saharan Africa: emergence of Candida auris as a major pathogen ", affiche présentée lors du Congrès européen de microbiologie clinique et des maladies infectieuses tenu du 10 au 13 mai 2014 à Barcelone, en Espagne). En Inde, C. auris a été mis en cause dans $5 \%$ des cas de septicémie à Candida survenus dans 27 unités de soins intensifs, mais certains établissements indiens déclarent des proportions allant de $17,5 \%$ à $30 \%(7,8)$. En date du 31 juillet 2018, les Centers for Disease Control and Prevention (CDC) des États-Unis ont déclaré 361 cas cliniques confirmés de C. auris dans des établissements de soins de santé américains; l'infection a été diagnostiquée chez 699 autres patients colonisés dans quatre États faisant l'objet d'une surveillance active (4). En Europe, au moins 120 cas de septicémie à Candida et 466 cas de colonisation sont apparus entre 2013 et 2017 (9).

Au Canada, les deux premiers patients infectés par $C$. auris avaient reçu des soins de santé en Inde $(1,10)$. Dans un cas, la caractérisation génomique du pathogène a suggéré que l'infection avait été contractée dans le sous-continent indien (11). L'importation d'autres cas est à prévoir. La transmission de l'infection dans les établissements de soins de santé canadiens est inévitable.

\section{Quelles sont les caractéristiques cliniques de la maladie causée par C. auris?}

Le spectre clinique de l'infection à $C$. auris va de la colonisation asymptomatique à la candidose invasive et se manifeste le plus souvent par une septicémie à Candida d'origine nosocomiale (12). Les infections du sang peuvent être de longue durée et difficiles à traiter; des taux bruts de mortalité d'environ 30 à $60 \%$ ont été déclarés $(5,13,14)$. Des complications métastatiques, comme la spondylodiscite, l'endocardite et la ventriculite, ont été décrites (13). Parmi les autres syndromes cliniques fréquemment déclarés figurent l'otomycose et l'otomastoïdite $(15,16)$. En fait, l'étymologie du nom auris reflète le siège anatomique d'où le premier isolat identifié a été prélevé, à savoir l'oreille d'un patient (17). L'infection a déjà touché d'autres sièges, y compris les voies respiratoires et urogénitales, l'abdomen ainsi que la peau et les tissus mous (18).

\section{Qui contracte l'infection par $C$. auris et comment l'infection se propage-t-elle?}

Les patients qui contractent une septicémie à Candida causée par $C$. auris ont habituellement des facteurs de risque en commun avec les patients atteints d'une maladie causée par d'autres espèces de Candida $(6,13,14,19)$. Ces facteurs comprennent l'hospitalisation, notamment dans une unité de soins intensifs, I'utilisation de cathéters veineux centraux, une intervention chirurgicale abdominale et l'exposition à des antibiotiques à large spectre ou à des antifongiques (20).

La pathogenèse de $C$. auris semble différente de celle des espèces classiques de Candida à plusieurs égards (tableau 1) (21). Outre l'espèce auris, la majorité des espèces de Candida importantes sur le plan clinique font partie des commensaux du tube digestif humain, à l'exception de C. parapsilosis,

Tableau 1 : Différences entre Candida auris et les espèces pathogènes classiques de Candida

\begin{tabular}{|c|c|c|}
\hline Caractéristique & Candida auris & $\begin{array}{c}\text { Espèces classiques } \\
\text { de Candida }\end{array}$ \\
\hline Habitat & Commensal de la peau & $\begin{array}{l}\text { Commensaux du tractus } \\
\text { gastro-intestinal }{ }^{\mathrm{b}}\end{array}$ \\
\hline Pathogenèse de l'infection & Exogène & Endogène \\
\hline Infections nosocomiales & Fréquente & Rare \\
\hline Contamination du milieu & Fréquente & Rare \\
\hline $\begin{array}{l}\text { Multirésistance aux } \\
\text { médicaments }\end{array}$ & Fréquente & Rare \\
\hline
\end{tabular}


qui colonise la peau (21). La pathogenèse de la septicémie à Candida causée par ces espèces est généralement le résultat d'une translocation de levures intestinales $(21,22)$; bien que la transmission nosocomiale de Candida soit parfois signalée, la maladie est le plus souvent causée par des souches qui font partie de la flore endogène du patient (23).

C'est surtout sur la peau des patients colonisés que se trouve C. auris, d'où le risque de contamination de l'environnement du patient et de transmission aux travailleurs de la santé et aux autres patients. De plus, les isolats de $C$. auris qui sont à l'origine des éclosions associées aux soins de santé entretiennent une relation clonale, ce qui suggère que la maladie est causée par des souches exogènes à propagation nosocomiale $(5,13,24,25)$.

\section{Quels sont les enjeux liés au diagnostic?}

C. auris peut être difficile à dépister à l'aide des épreuves de laboratoire courantes, ce qui peut retarder l'identification et l'isolement des patients colonisés ou infectés. Les épreuves d'identification biochimiques couramment utilisées dans les laboratoires de microbiologie clinique ne sont pas assez fiables pour identifier C. auris (26). Par exemple, C. auris peut être identifié à tort comme une autre espèce (généralement C. hæmulonii par le système VITEK-2 de bioMérieux,

Marcy-I'Étoile, en France [26], ou Rhodotorula glutinis, C. sake ou Saccharomyces cerevisiæ par le système API20CAUX [27]). Cette situation pourra être rectifiée au fur et à mesure que les bases de données des systèmes d'identification biochimiques seront mises à jour; par exemple, la carte VITEK-2 YST (v. 8.01) comprend maintenant $C$. auris.

C. auris peut être identifié avec précision par des appareils de spectrométrie de masse à temps de vol par désorption-ionisation laser assistée par matrice (Matrix Assisted Laser Desorption Ionisation -Time of Flight [MALDI-TOF]) dont les bases de données comprennent $C$. auris (il s'agit notamment des bases de données Bruker MALDI Biotyper CA et Research Use Only (RUO) les plus récentes, et de la base de données de bioMérieux VITEK MS RUO [v4.14 incluant la trousse Saccharomycetales]) et par des méthodes de séquençage moléculaire.

\section{Quels sont les enjeux liés au traitement?}

En général, les isolats de $C$. auris sont moins sensibles aux antifongiques que les autres espèces de Candida, mais les profils de sensibilité semblent être liés au clade géographique. La résistance au fluconazole est répandue sans toutefois être universelle comme on le craignait au départ (2); elle est maintenant considérée comme un facteur acquis plutôt que transmis (21). Les taux de résistance au fluconazole allaient de $14 \%$ parmi les isolats de Colombie (25) à plus de $90 \%$ parmi les isolats du clade sud-asiatique $(14,28)$; la résistance à l'amphotéricine $B$ et aux échinocandines semble également hétérogène. Plusieurs études ont fait état de taux de résistance à l'amphotéricine B d'environ $30 \%(5,14,25)$; Chowdhary et ses collaborateurs ont quant à eux déclaré une résistance à l'amphotéricine B dans $8 \%$ (27/350) des isolats indiens (28). Les taux de résistance à l'amphotéricine $B$ variaient significativement d'une région à l'autre de la Colombie (25). La résistance aux échinocandines est présente dans près de 2 à $5 \%$ des isolats $(5,28,29)$. Une résistance à deux classes d'antifongiques a été observée dans $41 \%$ des isolats testés à l'échelle mondiale (5). Rarement, les isolats peuvent être résistants aux trois principales classes d'antifongiques (5).

\section{Quels sont les enjeux en matière de prévention et de prise en charge des infections?}

Des éclosions nosocomiales sont à prévoir, car les patients peuvent rester colonisés ou leur milieu peut rester contaminé pendant des semaines, voire des mois, après une infection $(14,24,25,30)$. Des éclosions de grande envergure survenues dans des hôpitaux du Royaume-Uni ont été associées à I'utilisation de thermomètres axillaires à usages multiples (31); d'autres, survenues dans des hôpitaux d'Espagne, ont été associées à l'utilisation de brassards de tensiomètres (31). En outre, C. auris a été isolé à partir d'un large éventail de vecteurs passifs présents dans l'environnement des patients $(13,14,24,25)$. Les désinfectants cationiques de surface et les désinfectants à l'ammonium quaternaire sont inefficaces contre C. auris $(13,33,34)$. C. auris est aussi relativement résistant à l'effet des rayons ultraviolets (35). Le gluconate de chlorhexidine, la povidone iodée, l'eau de Javel et la vapeur d' $\mathrm{H}_{2} \mathrm{O}_{2}$ semblent efficaces contre C. auris (36).

Le rôle des travailleurs de la santé dans la propagation de C. auris est encore inconnu. Dans le cadre de l'enquête sur I'éclosion qui a sévi au Royaume-Uni, $C$. auris a été décelé dans les narines de 1/258 travailleurs de la santé; il s'agissait d'une infirmière qui prodiguait des soins à un patient fortement colonisé par cet agent (24). Pendant l'enquête sur une éclosion survenue en Colombie, C. auris a aussi été décelé sur les mains de deux travailleurs de la santé et sur l'aine d'un travailleur de la santé sur six. Le séquençage d'un génome entier a montré que ces souches étaient génétiquement identiques aux souches décelées chez un patient et dans son environnement (25).

Il peut s'avérer particulièrement difficile de surveiller et de limiter la propagation de $C$. auris en raison du transfert entre établissements de patients dont l'infection ou la colonisation par ce champignon est encore inconnue, ce qui augmente le risque de propagation de C. auris entre établissements (14). Par exemple, 112 patients d'hôpitaux et d'établissements de soins de longue durée de New York étaient infectés : 61 patients présentaient une septicémie à Candida et 51 autres étaient 
colonisés, d'après les épreuves de dépistage. Les patients infectés ou colonisés ont été transférés entre 24 hôpitaux et 24 établissements de soins de longue durée dans les 90 jours précédant la découverte de leur infection ou de leur colonisation (14).

\section{Répercussions sur les soins cliniques}

La reconnaissance, la prise en charge et l'isolement rapides des patients infectés ou colonisés par $C$. auris nécessitent la collaboration des hospitalistes/intensivistes, des microbiologistes, des experts en maladies infectieuses et des professionnels en prévention et prise en charge des infections.

Les cliniciens doivent savoir quelles méthodes d'identification des levures ont été utilisées par leur laboratoire de microbiologie local et envisager $C$. auris lorsque des espèces non identifiées ou inhabituelles de Candida sont isolées chez des patients qui ne répondent pas au traitement antifongique empirique (37). Il est recommandé de consulter un microbiologiste lorsque I'infection par C. auris est soupçonnée. Les isolats de C. auris suspects ou confirmés doivent être envoyés aux laboratoires provinciaux pour être soumis à d'autres analyses. Compte tenu des enjeux inhérents à la prévision des profils de sensibilité aux antifongiques, il est recommandé de soumettre tous les isolats cliniques de $C$. auris à des épreuves de sensibilité aux antifongiques. Le traitement de la maladie fongique doit être guidé par les résultats des épreuves de sensibilité aux antifongiques; les échinocandines conviennent néanmoins au traitement empirique en attendant les résultats de ces épreuves. Il est conseillé de consulter rapidement un spécialiste des maladies infectieuses. II n'est pas recommandé de traiter une colonisation asymptomatique.

La reconnaissance des patients chez qui une infection ou une colonisation par $C$. auris est soupçonnée ou confirmée justifie la consultation de professionnels locaux en prévention et prise en charge des infections. Les patients infectés ou colonisés doivent être isolés dans des chambres privées où sont adoptées les pratiques et précautions courantes contre les contacts; les chambres doivent être nettoyées quotidiennement avec des désinfectants sporicides. La question de savoir quand il convient de mettre fin aux précautions en matière d'isolement fait toujours l'objet de débats. Selon la recommandation actuelle des CDC, il faudrait soumettre les patients infectés ou colonisés à des tests périodiques de culture fongique par écouvillonnages mixtes de la région de l'aine et des aisselles pour vérifier si la colonisation persiste. À la suite de deux écouvillonnages de dépistage négatifs consécutifs, les patients pourraient être sortis de la chambre d'isolement (38). Dans la pratique, peu de cas déclarés ont répondu à ces critères (14). Par ailleurs, Public Health England recommande de maintenir les précautions en matière d'isolement pendant toute la durée de l'hospitalisation d'un patient (39). Cette recommandation s'explique en partie par le fait que les patients peuvent être colonisés à nouveau après un test négatif (Schelenz S. "Management of Candida auris outbreaks at a national level ", 20e congrès de I'International Society for Human and Animal Mycology tenu à Amsterdam, aux Pays-Bas, en juillet 2018).

Le tableau 2 présente un résumé de la marche à suivre pour déceler, évaluer et prendre en charge l'infection par C. auris. $D^{\prime}$ autres directives de prévention et de prise en charge des infections sont disponibles auprès des CDC (39).

Tableau 2 : Mesures à prendre pour le dépistage et la prise en charge de l'infection par Candida auris

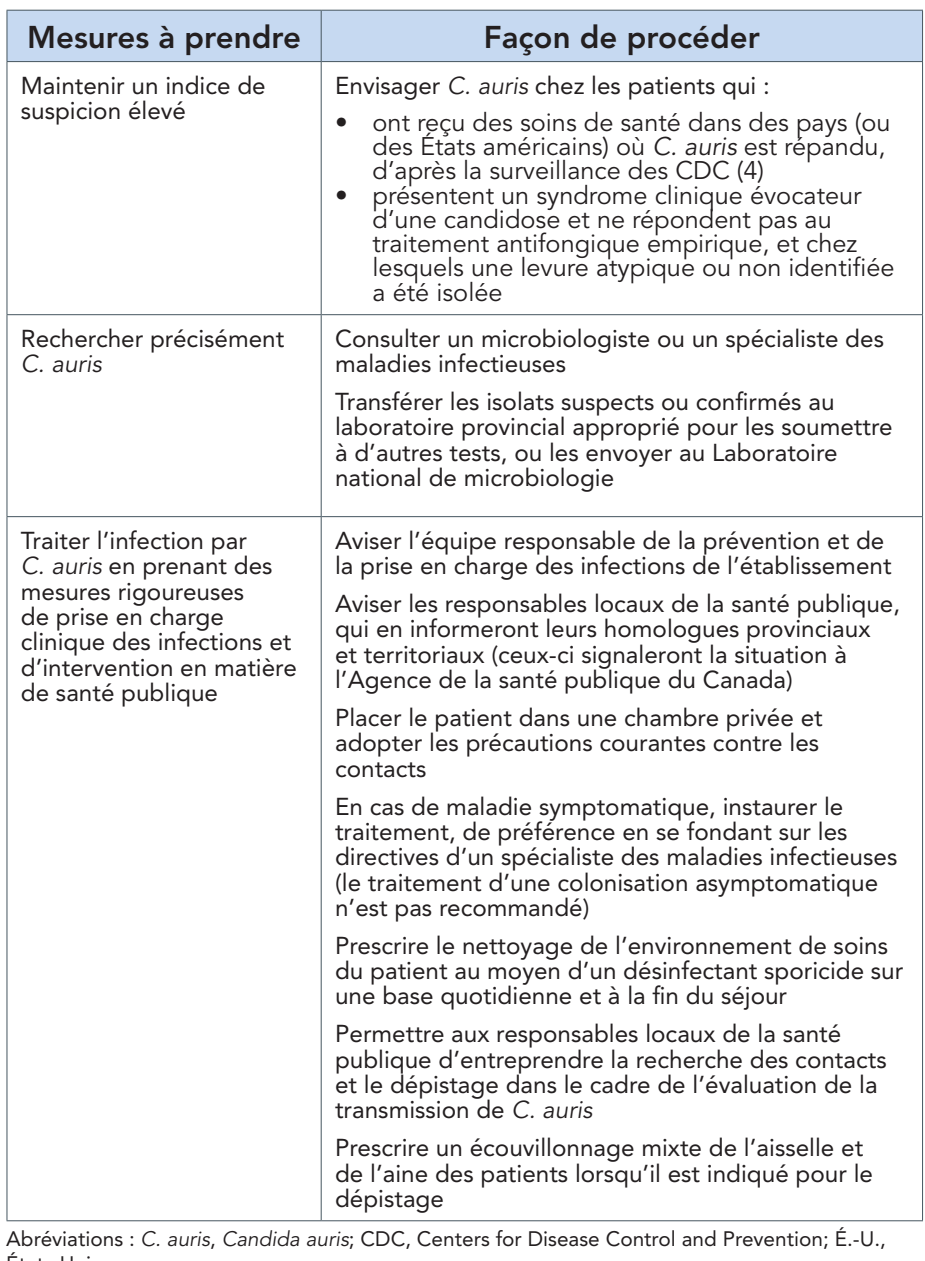

États-Unis

\section{Lacunes et étapes suivantes}

De nombreuses questions persistent quant à la meilleure façon de déceler $C$. auris et de limiter sa propagation au sein des établissements de santé canadiens et entre ces établissements. Il conviendrait de combler les lacunes en connaissances sur les méthodes optimales de dépistage en laboratoire et d'identification de $C$. auris en consolidant les bases de données biochimiques et d'identification MALDI-TOF existantes, et en élaborant des protocoles simples, rapides et sensibles de dépistage en laboratoire. Les incertitudes influant sur les pratiques de prévention et de prise en charge des infections à 
C. auris concernent la durée de la colonisation des patients (et ainsi, la durée recommandée l'isolement des patients après la première détection) et les stratégies de dépistage optimales. Par exemple, les épreuves de dépistage doivent-elles être réservées aux patients dont le contact avec un cas connu est avéré, ou doivent-elles être réalisées chez tous les patients ayant voyagé ou reçu des soins de santé dans des régions où $C$. auris est répandu? Étant donné que la répartition géographique de C. auris changera avec le temps et compte tenu des données de surveillance incomplètes issues de nombreuses régions, la reconnaissance des patients à risque élevé de colonisation par les travailleurs de la santé de première ligne peut être complexe.

Pour mieux comprendre l'épidémiologie de C. auris au Canada, les responsables du Programme canadien de surveillance des infections nosocomiales effectuent une surveillance nationale de l'infection dans les hôpitaux représentatifs de tout le pays. (Garcia-Jeldes F, Mitchell R, Bharat A, McGeer A pour le groupe d'intérêt à l'égard de C. auris du PCSIN. "Preparedness for Candida auris in Canadian Nosocomial Infection Surveillance Program [CNISP] Hospitals ", 2018, IDWeek 2018, du 3 au 7 octobre 2018, à San Francisco, en Californie). De plus, une étude de prévalence ponctuelle est prévue; elle visera à déterminer la prévalence de la colonisation et de l'infection dans les hôpitaux de soins tertiaires canadiens ( $D^{\text {re }}$ Allison McGeer, septembre 2018, communication personnelle). Les données sur la surveillance et la prévalence ponctuelle fourniront l'information nécessaire pour guider l'élaboration de politiques de prévention et de prise en charge des infections par ce pathogène émergent.

\section{Conflit d'intérêts}

Aucun.

\section{Références}

1. Schwartz IS, Hammond GW. Premier cas de Candida auris multirésistant au Canada. Relevé des maladies transmissibles au Canada 2017;43(7-8):168-72. DOI

2. Clancy CJ, Nguyen MH. Emergence of Candida auris: an international call to arms. Clin Infect Dis 2017 Jan;64(2):141-3. DOI PubMed

3. Lamoth F, Kontoyiannis DP. The Candida auris alert: facts and perspectives. J Infect Dis 2018 Jan;217(4):516-20. DOI PubMed

4. Centers for Disease Control and Prevention. Tracking Candida auris. Atlanta (GA): CDC; 2018. https://www.cdc. gov/fungal/diseases/candidiasis/tracking-c-auris.html

5. Lockhart SR, Etienne KA, Vallabhaneni S, Farooqi J, Chowdhary A, Govender NP, Colombo AL, Calvo B, Cuomo CA, Desjardins CA, Berkow EL, Castanheira M, Magobo RE, Jabeen K, Asghar RJ, Meis JF, Jackson B, Chiller T, Litvintseva AP. Simultaneous emergence of multidrug-resistant Candida auris on 3 continents confirmed by whole-genome sequencing and epidemiological analyses. Clin Infect Dis 2017 Jan;64(2):134-40. DOI PubMed
6. van Schalkwyk E, Shuping L, Ismail H, Thomas J, Govender NP. Independent risk factors associated with Candida auris candidaemia in South Africa - an analysis of national surveillance data, 2016-2017. South Afr J Infect Dis 2017;Supp: FIDSSA Oral Presentation Abstracts. https:// www.fidssa.co.za/content/images/fidssa_abstracts.pdf

7. Chowdhary A, Anil Kumar V, Sharma C, Prakash A, Agarwal K, Babu R, Dinesh KR, Karim S, Singh SK, Hagen F, Meis JF. Multidrug-resistant endemic clonal strain of Candida auris in India. Eur J Clin Microbiol Infect Dis 2014 Jun;33(6):919-26. DOI PubMed

8. Mathur P, Hasan F, Singh PK, Malhotra R, Walia K, Chowdhary A. Five-year profile of candidaemia at an Indian trauma centre: high rates of Candida auris blood stream infections. Mycoses 2018 May;61(9):674-80. DOl PubMed

9. Kohlenberg A, Struelens MJ, Monnet DL, Plachouras D; The Candida Auris Survey Collaborative Group. Candida auris: epidemiological situation, laboratory capacity and preparedness in European Union and European Economic Area countries, 2013 to 2017. Euro Surveill 2018 Mar;23(13): DOl PubMed

10. ProMED. Candida auris - Canada: (BC) ex India, coinfect. carbapenemase-pos. bacteria, VRE 20170923.5335411. Brookline (MA): International Society for Infectious Diseases; 2017 Sep 23. http://www. promedmail.org/direct. php?id=20170923.5335411

11. Bharat A, Tyler AD, Knox NC, Mabon P, Schwartz IS, Hammond GW, van Domselaar G, Antonation KS, Mulvey MR. Poster: Genomic characterization of the first multidrug-resistant Candida auris isolated in Canada. In: 14th ASM Conference on Candida and Candidiasis April 15-19, 2018, Providence (RI); 2018. https://www.asm.org/images/ ASM-Conferences/Candida/Poster\%20Session\%20B.pdf

12. Chowdhary A, Sharma C, Meis JF. Candida auris: A rapidly emerging cause of hospital-acquired multidrug-resistant fungal infections globally. PLoS Pathog 2017 May;13(5):e1006290. DOl PubMed

13. Ruiz-Gaitán A, Moret AM, Tasias-Pitarch M, Aleixandre-López Al, Martínez-Morel H, Calabuig E, Salavert-Lletí M, Ramírez P, López-Hontangas JL, Hagen F, Meis JF, Mollar-Maseres J, Pemán J. An outbreak due to Candida auris with prolonged colonisation and candidaemia in a tertiary care European hospital. Mycoses 2018 Jul;61(7):498-505. DOI PubMed

14. Adams E, Quinn M, Tsay S, Poirot E, Chaturvedi S, Southwick K, Greenko J, Fernandez R, Kallen A, Vallabhaneni S, Haley V, Hutton B, Blog D, Lutterloh E, Zucker H, Candida auris Investigation Workgroup. Candida auris in Healthcare Facilities, New York, USA, 2013-2017. Emerg Infect Dis 2018 Oct;24(10):1816-24. DOI PubMed

15. Choi HI, An J, Hwang JJ, Moon SY, Son JS. Otomastoiditis caused by Candida auris: case report and literature review. Mycoses 2017 Aug;60(8):488-92. DOI PubMed

16. Kim MN, Shin JH, Sung H, Lee K, Kim EC, Ryoo N, Lee JS, Jung SI, Park KH, Kee SJ, Kim SH, Shin MG, Suh SP, Ryang DW. Candida haemulonii and closely related species at 5 university hospitals in Korea: identification, antifungal 
susceptibility, and clinical features. Clin Infect Dis 2009 Mar;48(6):e57-61. DOI PubMed

17. Satoh K, Makimura K, Hasumi Y, Nishiyama Y, Uchida K, Yamaguchi $\mathrm{H}$. Candida auris sp. nov., a novel ascomycetous yeast isolated from the external ear canal of an inpatient in a Japanese hospital. Microbiol Immunol 2009 Jan;53(1):41-4. DOI PubMed

18. Jeffery-Smith A, Taori SK, Schelenz S, Jeffery K, Johnson EM, Borman A. Candida auris Incident Management Team, Manuel R, Brown CS. Candida auris: a review of the literature. Clin Microbiol Rev 2017 Nov;31(1):e00029-17. DOI PubMed

19. Rudramurthy SM, Chakrabarti A, Paul RA, Sood P, Kaur H, Capoor MR, Kindo AJ, Marak RSK, Arora A, Sardana R, Das S, Chhina D, Patel A, Xess I, Tarai B, Singh P, Ghosh A. Candida auris candidaemia in Indian ICUs: analysis of risk factors. J Antimicrob Chemother 2017 Jun;72(6):1794-801. DOI PubMed

20. Morales-López SE, Parra-Giraldo CM, Ceballos-Garzón A, Martínez HP, Rodríguez GJ, Álvarez-Moreno CA, Rodríguez JY. Invasive infections with multidrug-resistant yeast Candida auris, Colombia. Emerg Infect Dis 2017 Jan;23(1):162-4. DOI PubMed

21. Lockhart SR, Berkow EL, Chow N, Welsh RM. Candida auris for the clinical microbiology laboratory: not your grandfather's Candida species. Clin Microbiol News| 2017 Jul;39(13):99-103. DOI PubMed

22. Pappas PG, Lionakis MS, Arendrup MC, Ostrosky-Zeichner L, Kullberg BJ. Invasive candidiasis. Nat Rev Dis Primers 2018 May;4:18026. DOI PubMed

23. Pfaller MA. Nosocomial candidiasis: emerging species, reservoirs, and modes of transmission. Clin Infect Dis 1996 May;22 Suppl 2:S89-94. DOI PubMed

24. Schelenz S, Hagen F, Rhodes JL, Abdolrasouli A, Chowdhary A, Hall A, Ryan L, Shackleton J, Trimlett R, Meis JF, Armstrong-James D, Fisher MC. First hospital outbreak of the globally emerging Candida auris in a European hospital. Antimicrob Resist Infect Control 2016 Oct;5:35.

DOI PubMed

25. Escandón P, Chow NA, Caceres DH, Gade L, Berkow EL, Armstrong P, Rivera S, Misas E, Duarte C, Moulton-Meissner H, Welsh RM, Parra C, Pescador LA, Villalobos N, Salcedo S, Berrio I, Varón C, Espinosa-Bode A, Lockhart SR, Jackson BR, Litvintseva AP, Beltran M, Chiller TM. Molecular epidemiology of Candida auris in Colombia reveals a highly-related, country-wide colonization with regional patterns in Amphotericin B resistance. Clin Infect Dis 2018 May.DOI PubMed

26. Kathuria S, Singh PK, Sharma C, Prakash A, Masih A, Kumar A, Meis JF, Chowdhary A. Multidrug-resistant Candida auris misidentified as Candida haemulonii: characterization by matrix-assisted laser desorption ionization-time of flight mass spectrometry and DNA sequencing and its antifungal susceptibility profile variability by Vitek 2, CLSI broth microdilution, and Etest method. J Clin Microbiol 2015 Jun;53(6):1823-30. DOI PubMed

27. Mizusawa M, Miller H, Green R, Lee R, Durante M, Perkins R, Hewitt C, Simner PJ, Carroll KC, Hayden RT, Zhang SX.
Can multidrug-resistant Candida auris be reliably identified in clinical microbiology laboratories? J Clin Microbiol 2017 Feb;55(2):638-40. DOI PubMed

28. Chowdhary A, Prakash A, Sharma C, Kordalewska M, Kumar A, Sarma S, Tarai B, Singh A, Upadhyaya G, Upadhyay S, Yadav P, Singh PK, Khillan V, Sachdeva N, Perlin DS, Meis JF. A multicentre study of antifungal susceptibility patterns among 350 Candida auris isolates (2009-17) in India: role of the ERG11 and FKS1 genes in azole and echinocandin resistance. J Antimicrob Chemother 2018 Apr;73(4):891-9. DOl PubMed

29. Kordalewska M, Lee A, Park S, Berrio I, Chowdhary A, Zhao Y, Perlin DS. Understanding echinocandin resistance in the emerging pathogen Candida auris. Antimicrob Agents Chemother 2018 May;62(6):e00238-18. DOI PubMed

30. Welsh RM, Bentz ML, Shams A, Houston H, Lyons A, Rose LJ, Litvintseva AP. Survival, persistence, and isolation of the emerging multidrug-resistant pathogenic yeast Candida auris on a plastic health care surface. J Clin Microbiol 2017 Oct;55(10):2996-3005. DOI PubMed

31. Madder H, Moir I, Moroney R, Butcher L, Newnham R, Sunderland M, Clarke T, Foster D, Hoffman P, Moore G, Brown CS, Jeffery KJM. Multiuse patient monitoring equipment as a risk factor for acquisition of Candida auris. bioRxiv 2017:149054. DOI

32. Gordon M, Ruiz J, Villarreal E, Sáez Esteve I, Simó Martín C, Pérez Riera I, Gil LÁ, Castañeda Segura MJ, Ruiz A, Frasquet J, Peman Garcia J, Castellanos Á, Ramirez Galleymore P. First report of a textile environmental reservoir outbreak of Candida auris in a medical intensive care unit. In ECCMID April 22, 2018, Madrid (ES); 2018. http://www.eccmidlive. org/\#resources/first-report-of-an-textile-environmentalreservoir-during-an-outbreak-of-candida-auris-in-a-medicalintensive-care-unit

33. Kean R, Sherry L, Townsend E, McKloud E, Short B, Akinbobola A, Mackay WG, Williams C, Jones BL, Ramage G. Surface disinfection challenges for Candida auris: an in-vitro study. J Hosp Infect 2018 Apr;98(4):433-6. DOI PubMed

34. Cadnum JL, Shaikh AA, Piedrahita CT, Sankar T, Jencson $\mathrm{AL}$, Larkin EL, Ghannoum MA, Donskey CJ. Effectiveness of disinfectants against Candida auris and other Candida species. Infect Control Hosp Epidemiol 2017 Oct;38(10):1240-3. DOI PubMed

35. Cadnum JL, Shaikh AA, Piedrahita CT, Jencson AL, Larkin EL, Ghannoum MA, Ghannoum MA, Donskey CJ. Relative resistance of the emerging fungal pathogen Candida auris and other Candida species to killing by ultraviolet light. Infect Control Hosp Epidemiol 2018 Jan;39(1):94-6. DOl PubMed

36. Abdolrasouli A, Armstrong-James D, Ryan L, Schelenz S. In vitro efficacy of disinfectants utilised for skin decolonisation and environmental decontamination during a hospital outbreak with Candida auris. Mycoses 2017 Nov;60(11):75863. DOI PubMed

37. Lockhart SR, Jackson BR, Vallabhaneni S, Ostrosky-Zeichner L, Pappas PG, Chiller T. Thinking beyond the common Candida species: need for species-level identification of Candida due to the emergence of multidrug-resistant 
Candida auris. J Clin Microbiol 2017 Dec;55(12):3324-7. DOI PubMed

38. Centers for Disease Control and Prevention.

Recommendations for infection prevention and control for Candida auris. Atlanta (GA): CDC; 2018. https://www.cdc. gov/fungal/candida-auris/c-auris-infection-control.html
39. Public Health England. Guidance for the laboratory investigation, management and infection prevention and control for cases of Candida auris. London (UK): Public Health England; 2016 Jun 27 [mise à jour le 11 août 2017]. Report No.: 2013122. https://www.gov.uk/government/ publications/candida-auris-laboratory-investigationmanagement-and-infection-prevention-and-control

\section{Relevé des maladiestransmiscibles au Canac \\ RÉSUMÉ GRAPHIQUE \\ CANDIDA AURIS CE QUE LES POURVOYEURS DE SOINS DOIVENT SAVOIR}

Candida auris: un champignon multirésistant émergent

Il est maintenant au Canada

- il peut causer une maladie invasive

- il est difficile à détecter

- il se propage facilement

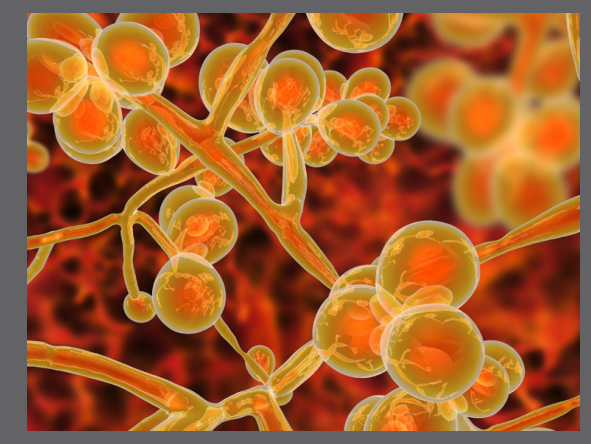

\section{Qui est à risque?}

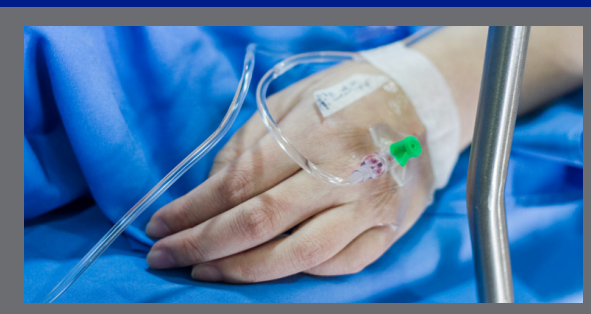

Ceux qui ne répondent pas au traitement antifongique et qui ont des antécédents de:

- soins de santé durant un voyage

- tests de laboratoire non spécifiques ou indiquant une espèce inhabituelle de candida

- cathéter veineux central

- chirurgie abdominale

- exposition à des antibiotiques ou à des antifongiques à large spectre

\section{Pratiques exemplaires}

Transférer le patient dans une chambre privée et consulter:

- un spécialiste des maladies infectieuses

- l'équipe de prévention et de contrôle des infections

- l'équipe de la santé publique

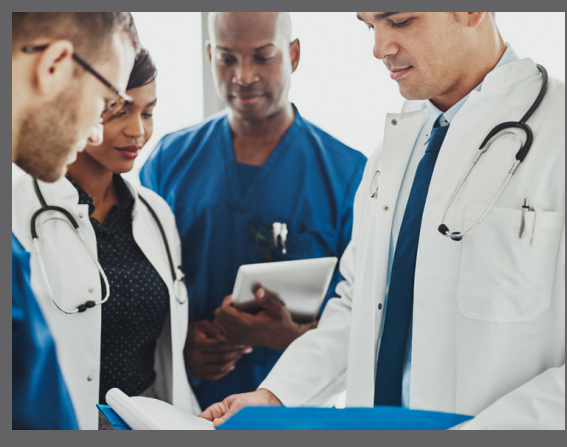

